\title{
The ${ }^{8} \mathrm{Li}+{ }^{2} \mathrm{H}$ reaction studied in inverse kinematics at 3.15 MeV/nucleon using the REX-ISOLDE post-accelerator
}

\author{
E. Tengborn, ${ }^{1}$ A. M. Moro, ${ }^{2}$ T. Nilsson, ${ }^{1,}{ }^{*}$ M. Alcorta, ${ }^{3}$ M. J. G. Borge, ${ }^{3}$ J. Cederkäll, ${ }^{4}$ C. Diget, ${ }^{5}$ L. M. Fraile, ${ }^{6}$ H. O. U. \\ Fynbo, ${ }^{5}$ J. Gomez-Camacho, ${ }^{2,7}$ H. B. Jeppesen, ${ }^{5}$ H. T. Johansson, ${ }^{1}$ B. Jonson, ${ }^{1}$ O. S. Kirsebom, ${ }^{5}$ H. H. Knudsen, ${ }^{5}$ \\ M. Madurga, ${ }^{3}$ G. Nyman, ${ }^{1}$ A. Richter, ${ }^{8,9}$ K. Riisager, ${ }^{5}$ G. Schrieder, ${ }^{8}$ O. Tengblad,${ }^{3}$ N. Timofeyuk, ${ }^{10}$ M. Turrion, ${ }^{3}$ \\ D. Voulot, ${ }^{11}$ and F. Wenander ${ }^{11}$ \\ ${ }^{1}$ Fundamental Fysik, Chalmers Tekniska Högskola, S-412 96 Göteborg, Sweden \\ ${ }^{2}$ Departamento de FAMN, Universidad de Sevilla, Apdo. 1065, E-41080 Sevilla, Spain \\ ${ }^{3}$ Instituto Estructura de la Materia, CSIC, E-28006 Madrid, Spain \\ ${ }^{4} \mathrm{PH}$ Department, CERN, CH-1211 Genève 23, Switzerland \\ ${ }^{5}$ Department of Physics and Astronomy, University of Aarhus, DK-8000 Aarhus C, Denmark \\ ${ }^{6}$ Grupo de Física Nuclear, Universidad Complutense, E-28040 Madrid, Spain \\ ${ }^{7}$ Centro Nacional de Aceleradores, Avda. Thomas A. Edison, E-41092, Sevilla, Spain \\ ${ }^{8}$ Institut für Kernphysik, Technische Universität, D-64289 Darmstadt, Germany \\ ${ }^{9}$ ECT*, Villa Tambosi, I-38123 Villazzano (Trento), Italy \\ ${ }^{10}$ Department of Physics, University of Surrey, Guildford, Surrey GU2 7XH, UK \\ ${ }^{11}$ AB Department, CERN, CH-1211 Genève 23, Switzerland \\ (Received 10 September 2011; published 27 December 2011)
}

\begin{abstract}
The reaction ${ }^{8} \mathrm{Li}+{ }^{2} \mathrm{H}$ has been studied in inverse kinematics at the incident energy of $3.15 \mathrm{MeV} /$ nucleon, using the REX-ISOLDE post-accelerator. The reaction channels corresponding to $(d, p),(d, d)$, and $(d, t)$ reactions populating ground states and low-lying excited states in ${ }^{7-9} \mathrm{Li}$ have been identified and the related angular distributions extracted and compared with coupled-channels, distorted-wave Born approximation (DWBA), and coupled-reaction-channels calculations. For the inelastic and $(d, t)$ channels we find that higher order effects are very important and hence one needs to go beyond the simple DWBA to extract reliable structure information from these processes.
\end{abstract}

DOI: 10.1103/PhysRevC.84.064616

\section{INTRODUCTION}

The method of transfer reactions using light projectiles on heavy targets has a long history as a spectroscopic tool to investigate nuclear structure. The possibility of using the angular distributions to extract the angular momentum transfer gives the opportunity to deduce spins and parities of the populated states, as well as spectroscopic factors if the cross section can be obtained on an absolute scale. Thus, there are many arguments to try to enlarge the scope of transfer reactions also to nuclei far from stability, to gain insight into the structural changes at and beyond the drip lines. Consequently, transfer reaction experiments are performed at the majority of existing facilities that have access to low-energy radioactive ion beams (see, e.g., [1-7] and references therein). However, the combination of having to utilize low-intensity radioactive beams in inverse kinematics geometry with the loosely bound states involved complicates both the experiment and the interpretation. Several of these challenges are summarized in Refs. [8-10].

The current work is part of a series of nucleon transfer reaction experiments that have been performed to study the neutron-rich lithium isotopes with the post-accelerator REXISOLDE at CERN-ISOLDE. Earlier results from experiments using a ${ }^{9} \mathrm{Li}$ beam at $2.36 \mathrm{MeV} /$ nucleon impinging on a deuter-

\footnotetext{
*thomas.nilsson@chalmers.se
}

PACS number(s): 24.10.Eq, 24.50.+g, 25.60.Bx, 25.45.De

ated polyethylene target have been presented in Refs. [11-14]. The analysis in Ref. [14] concentrated on the $(d, t)$ channel where it was shown that all previously known states in ${ }^{8} \mathrm{Li}$ below $4 \mathrm{MeV}$ excitation energy were populated. From the extracted excitation energies in ${ }^{8} \mathrm{Li}$ and the angular distribution of the tritons, spectroscopic factors were deduced for the ground state and first two excited states. The experimental data were compared to distorted-wave Born approximation (DWBA) calculations using spectroscopic factors from both shell-model calculations as well as $a b$ initio calculations using the quantum Monte Carlo model. The simple DWBA calculations underestimate the experimental cross section but reproduce the shape of the angular distribution; see, e.g., Fig. 3 in Ref. [14]. Two sets of optical model (OM) potential parameters gave similar underestimations. To explain the observed deficiency, a possible contribution from compound nucleus formation was estimated with the TALYS code [15]. Although generic Hauser-Feshbach codes normally are not very reliable for light nuclei, an upper limit on the compound contribution could be extracted by investigating large scattering angles since the resulting angular distributions are largely isotropic in the center-of-mass system. By assuming such a contribution together with the DWBA part, the experimental angular distributions were well described for the ground state and the first excited state. However, the spectroscopic factors had to be scaled by approximately a factor of 2 to reproduce the absolute cross sections, indicating that the model assumptions were too simple. 
In a similar experiment done at TRIUMF using a ${ }^{9} \mathrm{Li}$ beam of $1.68 \mathrm{MeV} /$ nucleon [16], the distributions from DWBA calculations were only fitted to data taken under small centerof-mass angles where the compound nuclear contribution to the cross section has a negligible effect.

This observation motivated us to perform a benchmark experiment with a ${ }^{8} \mathrm{Li}$ beam on the same target to study the reaction ${ }^{2} \mathrm{H}\left({ }^{8} \mathrm{Li}, p\right){ }^{9} \mathrm{Li}^{*}$ and thereby test the validity of the analysis methods. Other reaction channels simultaneously present are the elastic/inelastic scattering channels, ${ }^{2} \mathrm{H}\left({ }^{8} \mathrm{Li}, d\right){ }^{8} \mathrm{Li}^{*}$, and the one-neutron stripping reaction, ${ }^{2} \mathrm{H}\left({ }^{8} \mathrm{Li}, t\right){ }^{7} \mathrm{Li}^{*}$.

The paper is organized as follows: In Sec. II we describe the experimental setup. In Sec. III, the measured elastic, inelastic, and transfer data are compared with optical model, DWBA, and coupled-channels calculations. Finally, in Sec. IV we summarize the main conclusions of this work.

\section{EXPERIMENTAL CONDITIONS}

The experiment was performed at the ISOLDE facility [17] at CERN using the post-accelerator REX-ISOLDE [18]. A ${ }^{8} \mathrm{Li}^{1+}$ beam was produced by bombarding a Ta-foil target with a pulsed $1.4-\mathrm{GeV}$ proton beam from the PS Booster in combination with a tungsten hot-surface ion source. The beam was then mass separated using the High Resolution Separator and steered to REX-ISOLDE. At REX-ISOLDE the $1^{+}$ions were first bunched in REXTRAP and subsequently charge bred in the REX-EBIS ion source [19] to $2^{+}$, i.e., $A / Q=4$. This implied a background from both ${ }^{12} \mathrm{C}^{3+}$ and ${ }^{16} \mathrm{O}^{4+}$ from residual gases in the REX-EBIS source. The ions were accelerated to $3.15 \mathrm{MeV} /$ nucleon in the REX-ISOLDE LINAC. In order to avoid the carbon component in the beam, a stripping foil was introduced in front of the last dipole magnet where a setting of $A / Q=8 / 3$ was applied to select ${ }^{8} \mathrm{Li}^{3+}$, yielding an average intensity of $1.6 \times 10^{6}$ ions $/ \mathrm{s}$. This mass-to-charge ratio excluded all charge states of carbon whereas a contamination of ${ }^{16} \mathrm{O}^{6+}$ still was present, albeit the level was reduced. In addition to the measurements under these conditions, background measurements without production of ${ }^{8} \mathrm{Li}$, i.e., with a beam predominantly consisting of ${ }^{16} \mathrm{O}^{6+}$ at the same energy, were performed.

The experimental setup, with two telescope detectors, is shown in Fig. 1. A collimator with a $2-\mathrm{mm}$ aperture defined the radioactive beam hitting one of four reaction targets placed on a target ladder, at an angle of $22^{\circ}$ with respect to the beam axis. The targets were deuterated polyethylene (with a thickness of $9.8 \mu \mathrm{m})$, polyethylene $(12.7 \mu \mathrm{m}),{ }^{109} \mathrm{Ag}(0.92 \mu \mathrm{m})$, and ${ }^{12} \mathrm{C}$ (7.7 $\mu \mathrm{m})$ foils.

Two double-sided silicon-strip detectors (DSSSD) were used as $\Delta E$ detectors, having $32 \times 32$ strips and an active area of $6.4 \times 6.4 \mathrm{~cm}^{2}$. The energy loss in the dead layer was treated as described in Ref. [20]. The thickness of the $\Delta E$ detector was $61 \mu \mathrm{m}$ in the forward direction and $62 \mu \mathrm{m}$ in the backward direction. The $E$ detectors had the same area as the $\Delta E$ detectors and a thickness of $1500 \mu \mathrm{m}$, with no segmentation. The two telescopes were mounted in the forward and backward directions, respectively, in order to cover as wide an angular range as possible, $\theta_{\mathrm{lab}} \in\left[13^{\circ}, 175^{\circ}\right]$. In the beam

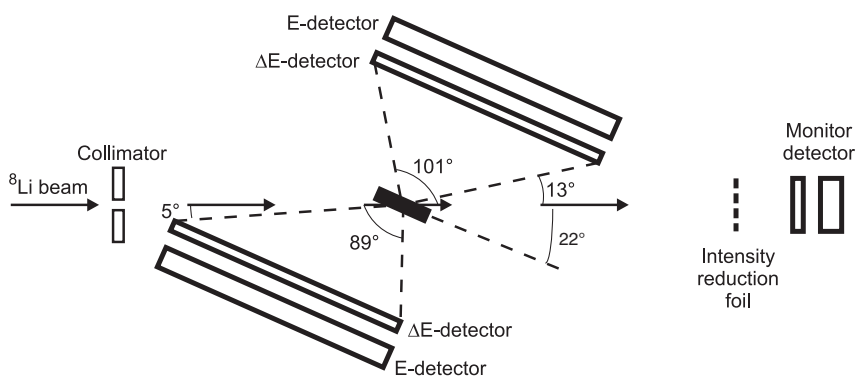

FIG. 1. Sketch of the experimental setup (not to scale).

dump, $30 \mathrm{~cm}$ from the target, a telescope detector referred to as the monitor detector was mounted in order to watch the relative beam intensity and its isotopic admixture. This detector could not directly be used for an absolute normalization since the direct beam intensity would have damaged the detectors. To avoid this, several micro-perforated GEM foils were placed in front of the detector in order to mechanically decrease the beam intensity, which however introduced an ill-determined transmission factor that had to be determined in the analysis process as described in the following section.

\section{ANALYSIS AND RESULTS}

Particle identification in the forward telescope was made using the $\Delta E$ versus $E$ information. Figure 2 shows the separation of protons, deuterons, tritons, and $\alpha$ particles. Particle identification is not possible in the backward telescope due to the low energies of ejectiles in this kinematical domain, implying that only few particles pass through the $\Delta E$ detector and enter the $E$ detector. The absolute number of incoming ions had to be determined by relating the rates in the monitor telescope to the beam intensity. This was made using data taken with the aforementioned silver target where an angular distribution of Rutherford-scattered ${ }^{8} \mathrm{Li}$ could be identified. This procedure introduces a systematic error of $\sim 10 \%$ in all absolute cross sections. The same dataset was also used to determine the detector positions with respect to the beam axis.

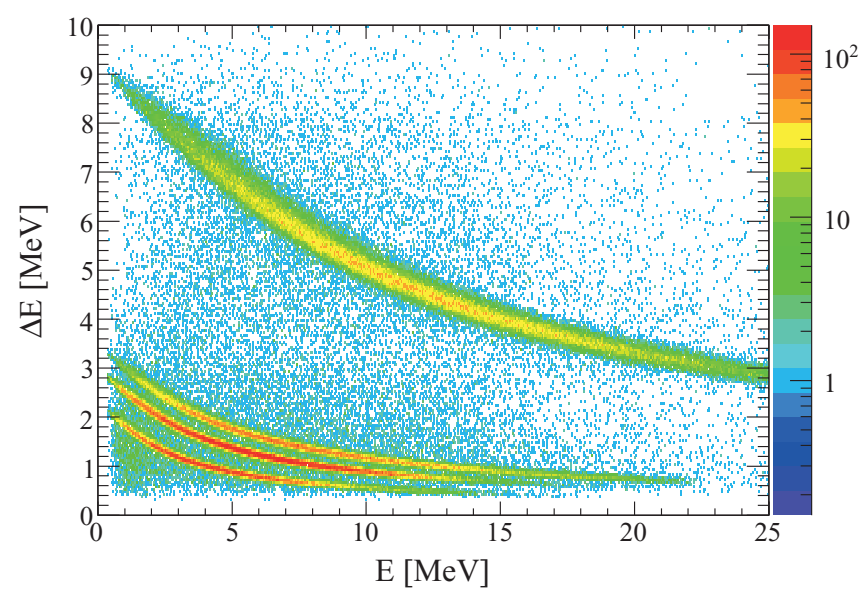

FIG. 2. (Color online) $\Delta E$ vs $E$ plot for all particles registered in the forward telescope detector showing the identification of protons, deuterons, tritons, and $\alpha$ particles. 


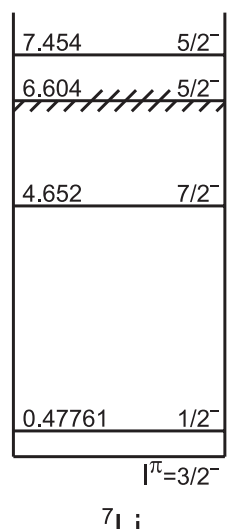

${ }^{7} \mathrm{Li}$
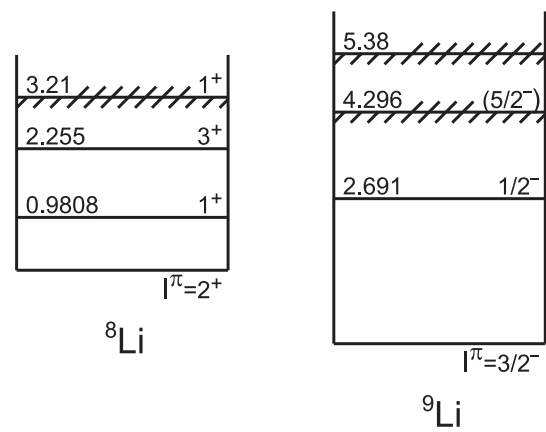

FIG. 3. Level schemes for ${ }^{7} \mathrm{Li}[21],{ }^{8} \mathrm{Li}$ [22], and ${ }^{9} \mathrm{Li}$ [22] (with energies in $\mathrm{MeV}$ ) showing only the low-lying levels populated in the present experiment with a ${ }^{8} \mathrm{Li}$ beam. For ${ }^{8} \mathrm{Li}$ the observed levels are the same as in the experiment using a ${ }^{9} \mathrm{Li}$ beam [11-14].

Light ejectiles could also be stemming from reactions involving the ${ }^{16} \mathrm{O}$ component in the beam, or ${ }^{8} \mathrm{Li}$ reacting with the carbon nuclei in the deuterated polyethylene. The signal to background ratio for the former contribution was optimized in the data analysis by applying a time cut of $800 \mathrm{~ms}$ after the impact of the pulsed proton beam (having a typical time interval of $2.4 \mathrm{~s}$ ), which still selects $66 \%$ of the post-accelerated ${ }^{8} \mathrm{Li}$. The remaining contributions from the ${ }^{16} \mathrm{O}$ component $(1.5 \%)$ were investigated using the background runs without radioisotope production and the reactions on carbon by using a dataset taken with a pure carbon target. After correct scaling factors were applied, these contributions were subtracted from all excitation energy spectra as well as angular distributions.

Figure 3 shows the energy level schemes of ${ }^{7} \mathrm{Li}$ [21], ${ }^{8} \mathrm{Li} \mathrm{[22],} \mathrm{and}{ }^{9} \mathrm{Li}$ [22] for the states being populated in the experiment. The reactions populating these levels are identified by the observed combination of kinetic energy and the laboratory angle of the corresponding ejectile. By using the unambiguously identified protons in the forward direction, the population of the ground state as well as the first three excited states in ${ }^{9} \mathrm{Li}$ is observed. The kinematical curves for ejectiles corresponding to the two lowest-lying states exceed the detection threshold also for backward angles of the setup due to the positive $Q$ value for the ${ }^{2} \mathrm{H}\left({ }^{8} \mathrm{Li}, p\right)^{9} \mathrm{Li}$ reaction, $Q=1.839 \mathrm{MeV}$. However, for reactions populating the first excited state in ${ }^{9} \mathrm{Li}$, these events are too close to threshold for a reliable assignment without unambiguous particle identification. In contract, the reactions populating the ${ }^{9} \mathrm{Li}$ ground state can be reliably assigned also without particle identification; since this region is kinematically forbidden for most other channels, and the intensity largely follows the expected kinematical curve as shown in Fig. 4, all these events are assumed to be protons. It was not possible to follow such a procedure for the other ejectiles.

For the particles identified as deuterons, the ground state and the three first excited states in ${ }^{8} \mathrm{Li}$ are observed, and

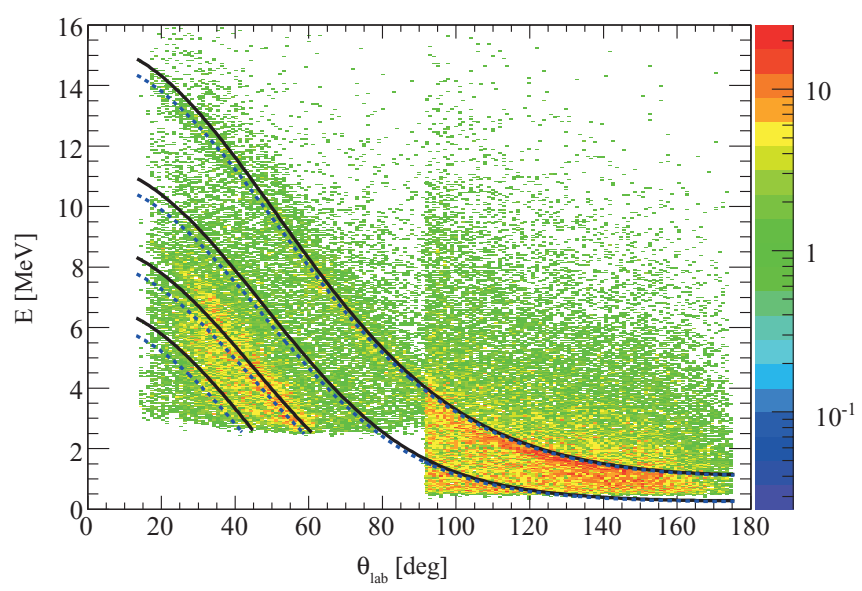

FIG. 4. (Color online) The total energy deposited in the telescope detectors as a function of the laboratory angle $\theta_{\text {lab }}$ for protons. For deuterons and tritons data are only registered in the telescope detector in the forward direction due to kinematical constraints. The groups of overlaid kinematical curves represent feeding to the known low-lying states in ${ }^{9} \mathrm{Li}$. In each group, the curves correspond to reactions in the front (black) and back (dashed blue line) of the target.

for the particles identified as tritons, the ground state $(Q=$ $4.225 \mathrm{MeV}$ ) and the first four excited states in ${ }^{7} \mathrm{Li}$ are observed. For the latter reaction channel, the ground state and first excited state at $0.48 \mathrm{MeV}$ cannot be separated due to the limited resolution induced by the granularity of the setup and the beam spread.

The corresponding excitation energy spectra for the identified reaction channels are shown in Fig. 5 without any correction for the experimental acceptance. The reaction is assumed to take place in the center of the target. The energy of excited levels agree well with the literature values shown in Fig. 3. The relative weights of the fits to the ground state and the first excited state in Fig. 5(c) are not conclusive since, as mentioned above, these states could not be well resolved.

This type of target is known to have a residual ${ }^{1} \mathrm{H}$ component, permitting ${ }^{1} \mathrm{H}\left({ }^{8} \mathrm{Li}, p\right)$ scattering. The thus ejected protons can, at small laboratory angles, approximately fulfill the same kinematical conditions as protons stemming from the ${ }^{2} \mathrm{H}\left({ }^{8} \mathrm{Li}, p\right){ }^{9} \mathrm{Li}$ reaction populating the state at $4.296 \mathrm{MeV}$ in ${ }^{9} \mathrm{Li}$. Within the acceptance of the current experiment, this would appear as a contribution peaked at $\sim 3.9 \mathrm{MeV}$ in the excitation energy spectrum shown in Fig. 5(a). Thus, given the small ${ }^{1} \mathrm{H}$ fraction ( $\sim 3 \%$ as estimated in Ref. [12]), and the fact that the excitation energy spectrum can be described by known states from Ref. [22], we conclude that there is no sizable contribution from scattering on residual ${ }^{1} \mathrm{H}$ in the data.

Following acceptance corrections and the previously described background-subtraction procedures, absolute differential cross sections have been extracted for all reaction channels and are presented according to normal kinematics, i.e., with a light particle impinging on a heavy target. As mentioned, the $Q$ value for populating the ground state in ${ }^{9} \mathrm{Li}$ is high, meaning that large scattering angles of the ejectile in the laboratory system could be detected, corresponding to small angles in the center-of-mass system. This allows for measurements within a large range of scattering angles for this particular channel. 


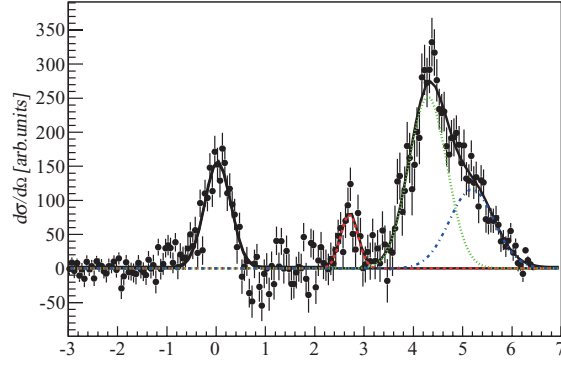

(a) Protons

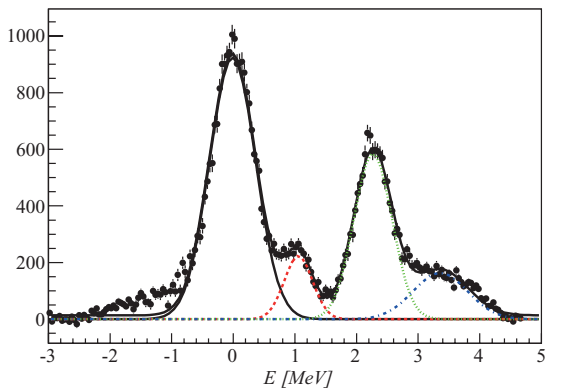

(b) Deuterons

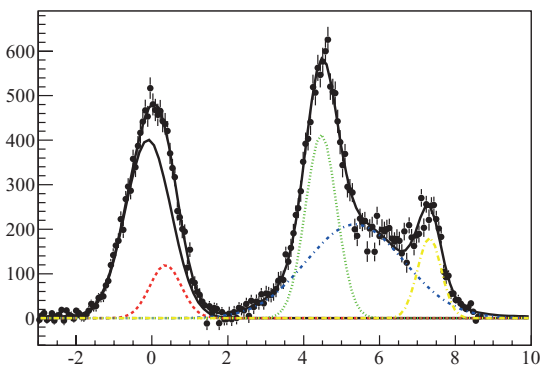

(c) Tritons

FIG. 5. (Color online) Excitation energy spectra for the reactions ${ }^{2} \mathrm{H}\left({ }^{8} \mathrm{Li}, p\right) \mathrm{X}(\mathrm{a}),{ }^{2} \mathrm{H}\left({ }^{8} \mathrm{Li}, d\right) \mathrm{X}(\mathrm{b})$, and ${ }^{2} \mathrm{H}\left({ }^{8} \mathrm{Li}, t\right) \mathrm{X}(\mathrm{c})$ with subtraction of contributions from the ${ }^{16} \mathrm{O}$ contamination as well as ${ }^{8} \mathrm{Li}$ reacting with carbon in the deuterated polyethylene target. The data are given in arbitrary units since no acceptance correction was attempted here. The relative weights of the fits to the ground state and the first excited state in (c) are not conclusive since these states could not be well resolved by the experimental setup.

Theoretical calculations of the different open reaction channels have been performed using the coupled-channels code FRESCO [23]. The results of these calculations are presented in the next sections.

\section{A. Analysis of the elastic scattering data}

Due to the inherent experimental limitations of the inverse kinematics, the elastic scattering cross section could only be measured within the angular range $\theta_{\text {c.m. }}=60^{\circ}-145^{\circ}$ (in normal kinematics). When plotted relative to the Rutherford cross section (see Fig. 6), the angular distribution displays a broad maximum around $115^{\circ}$. Optical model calculations performed with different deuteron optical potentials taken from the literature $[12,24]$ predict another maximum around $35^{\circ}$. Thus, due to the lack of data at these forward angles, the determination of physical meaningful set of parameters is subject to a large ambiguity. To reduce this ambiguity, we have calculated the real part of the $d+{ }^{8} \mathrm{Li}$ potential by means of a double-folding (DF) procedure, in which the deuteron and ${ }^{8} \mathrm{Li}$ densities are convoluted with an effective NN interaction,

$V^{\mathrm{DF}}(R)=\int d \mathbf{r}_{d} \int d \mathbf{r}_{p} \rho_{d}\left(r_{d}\right) \rho_{\mathrm{Li}}^{(0)}\left(r_{p}\right) v_{N N}\left(\left|\mathbf{R}-\mathbf{r}_{d}+\mathbf{r}_{p}\right|\right)$,

where $\rho_{d}\left(r_{d}\right)$ and $\rho_{\mathrm{Li}}^{(0)}\left(r_{p}\right)$ denote the deuteron and ${ }^{8} \mathrm{Li}$ ground state densities, respectively, and $v_{N N}$ is the effective NN interaction. For the latter we used the spin-isospin independent part of the M3Y interaction based on the Reid soft-core NN potential [25], supplemented by the pseudopotential simulating nucleon knockout exchange [26]. Following [27], the deuteron density is calculated from the Hulthén wave function. We used the ${ }^{8} \mathrm{Li}$ density calculated in Ref. [28] within the microscopic three-cluster model ${ }^{8} \mathrm{Li}=\alpha+t+n$. The $\mathrm{DF}$ potential was calculated with the code DFPOT [29].

This DF potential is supplemented with an imaginary part $\left[W_{d}(R)\right]$ for which we adopt a Woods-Saxon derivative form. A spin-orbit term, coupling the deuteron spin to the projectiletarget relative orbital angular momentum, was also included. Its radial part has the usual Woods-Saxon derivative form, with

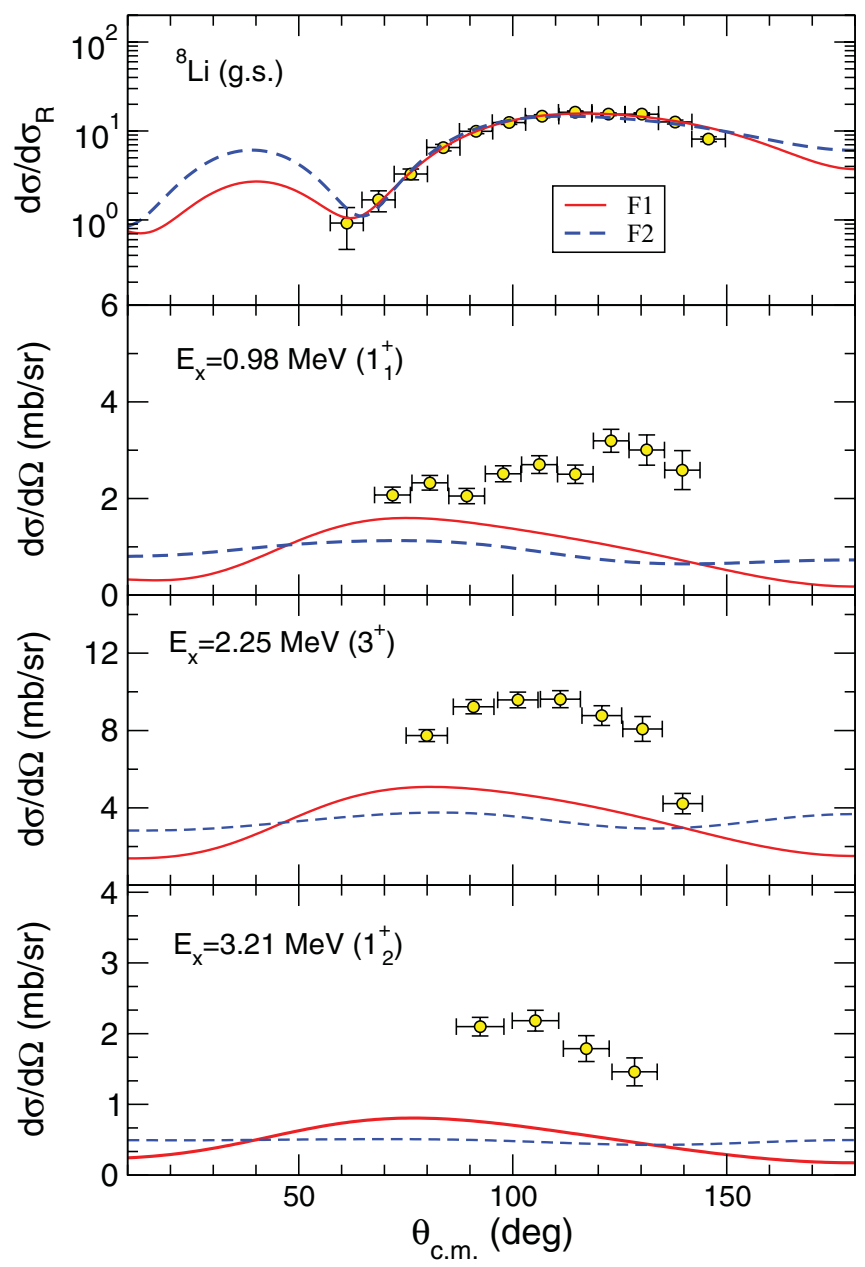

FIG. 6. (Color online) Angular dependence of differential cross sections for the elastic and inelastic reaction channels, detecting an outgoing deuteron. The curves in the top panel are optical model calculations with the parameters described in the text. In the other panels, they represent DWBA calculations using doublefolding coupling potentials with microscopic transition densities. The horizontal and vertical error bars in the experimental data denote bin widths and purely statistical errors, respectively. 
TABLE I. Parameters for the $d+{ }^{8} \mathrm{Li}$ potentials used in this work. The quantities $J_{r}$ and $J_{i}$ denote the real and imaginary volume integrals, divided by the product of the projectile and target masses.

\begin{tabular}{|c|c|c|c|c|c|c|c|c|c|c|}
\hline $\mathrm{OM}(\mathrm{F} 1)$ & 0.72 & 9.7 & 1.55 & 0.31 & 6.9 & 1.20 & 0.90 & 296 & 93 & 805 \\
\hline $\mathrm{CC}(\operatorname{set} 1)$ & 0.73 & 16.7 & 1.58 & 0.20 & 7.0 & 1.76 & 0.30 & 300 & 106 & 705 \\
\hline $\mathrm{CC}(\operatorname{set} 2)$ & 1 & 8.0 & 1.67 & 0.20 & 0 & - & - & 411 & 56 & 885 \\
\hline
\end{tabular}

reduced radius $r_{s o}=1.2 \mathrm{fm}$ and diffuseness $a_{s o}=0.90 \mathrm{fm}$, taken from [30]. The normalization of the real part $\left(N_{r}\right)$, the parameters of the surface Woods-Saxon potential $\left(W_{d}, r_{i}, a_{i}\right)$, and the depth of the spin-orbit term $\left(V_{s o}\right)$ were allowed to vary to best fit the elastic scattering data. This yields the values listed in Table I (potential F1 hereafter). Along with the potential parameters, the volume integral of the real $\left(J_{r}\right)$ and imaginary $\left(J_{i}\right)$ parts and the reaction cross section are also provided. The sizable renormalization of the real part $\left(N_{r}=0.72\right)$ is attributed to the dynamical effects arising from nonelastic processes, such as target excitation or deuteron breakup. The calculated elastic cross section angular distribution, relative to the Rutherford cross section, is shown by the solid line in the upper panel of Fig. 6.

Anticipating the discussion of Sec. III C, we have found that the magnitude and shape of the ${ }^{8} \mathrm{Li}(d, p){ }^{9} \mathrm{Li}$ cross section depend strongly on the choice of the deuteron optical potential used in the DWBA calculations. In particular, we have noticed that in order to get the correct shape of the $\left(d, p_{0}\right)$ angular distribution when populating the ${ }^{9} \mathrm{Li}$ ground state, the imaginary part requires a large reduced radius $\left(r_{i} \approx 2 \mathrm{fm}\right)$. Interestingly, this result has been also reported by Powell et al. [30] in their analysis of $(d, p)$ data with several lithium and beryllium isotopes. Therefore, we have considered a second set of parameters, in which we fix the radius of the imaginary potential to the value used in Ref. [30], $r_{i}=2.1 \mathrm{fm}$, and fit the remaining parameters. This gives the values listed in Table I and labeled F2. Again, the real part requires a significant renormalization to account for the data $\left(N_{r}=0.57\right)$. The calculated elastic scattering angular distribution is shown by the dashed line in the upper panel of Fig. 6.

\section{B. Analysis of the inelastic scattering data}

In the excitation energy spectrum of ${ }^{8} \mathrm{Li}$ [see Fig. 5(b)] the population of the first excited state $\left(E_{x}=0.98 \mathrm{MeV}\right.$, $\left.1^{+}\right)$and the resonances at $E_{x}=2.25 \mathrm{MeV}\left(3^{+}\right)$and $E_{x}=$ $3.21 \mathrm{MeV}\left(1^{+}\right)$are clearly seen. In this section we present a joint analysis of the elastic and inelastic channels within the DWBA and coupled-channels (CC) methods. To allow for the ${ }^{8} \mathrm{Li}$ excitation, one needs the coupling potentials between the ground state and the excited states. These potentials are generated microscopically from the corresponding transition densities by a generalization of Eq. (1),

$$
V_{i j}^{(\lambda)}(R)=\int d \mathbf{r}_{d} \int d \mathbf{r}_{p} \rho_{d}\left(r_{d}\right) \rho_{i j}^{(\lambda)}\left(r_{p}\right) v_{N N}\left(\left|\mathbf{R}-\mathbf{r}_{d}+\mathbf{r}_{p}\right|\right),
$$

where $\rho_{i j}^{(\lambda)}\left(r_{p}\right)$ is the ${ }^{8} \mathrm{Li}$ transition density between states $i$ and $j$ and multipolarity $\lambda$. The densities for the $1_{2}^{+}$state at 3.21 MeV are not reported in Ref. [28]. Given that the transition densities for the other states are similar in shape, and differ mostly in their magnitude, we have assumed that the transition densities for the $1_{2}^{+}$state have same radial behavior as the $1_{1}^{+}$ state, but keeping in mind that these densities might be affected by a normalization factor.

Following the approach used in previous microscopic CC analyses (see, e.g., [27,31]) the transition potentials include also an imaginary part, calculated as the derivative of the monopole central potential, multiplied by a reduced matrix element of the deformation length operator (for shortness, deformation lengths hereafter), i.e.,

$$
U_{i j}^{(\lambda)}(R)=N_{r} V_{i j}^{(\lambda)}(R)+\imath \delta_{i f}^{\lambda} \frac{d W_{d}(R)}{d R},
$$

where $\delta_{i f}^{\lambda}$ is the deformation length between the states $i$ and $j$. We include both diagonal $(i=j)$ as well as nondiagonal couplings. The deformation lengths are obtained from the transition densities as ${ }^{1}$

$$
\delta_{i f}^{\lambda} \equiv\left\langle I_{f}\left\|\hat{\delta}_{\lambda}\right\| I_{i}\right\rangle=\frac{\sqrt{2 I_{f}+1}}{\lambda+2} \frac{\int \rho_{i f}^{(\lambda)}(r) r^{\lambda+2} d r}{\int \rho_{\mathrm{Li}}^{(0)}(r) r^{\lambda+1} d r} .
$$

The results of these DWBA calculations are compared in Fig. 6 with the experimental angular distributions. The solid and dashed lines correspond to the deuteron optical potentials F1 and F2, respectively. In both cases, the calculation fails to reproduce the shape and magnitude of the data, largely underpredicting the measured inelastic cross section.

A possible reason for the discrepancy between the calculations and the data could be the inadequacy of the DWBA method for the present case. This method relies on the assumption that the inelastic cross section is small compared to the elastic one. However, given the large yield for the population of the $3^{+}$resonance at $2.5 \mathrm{MeV}$ [see Fig. 5(b)] this does not seem to be the present case. Under these circumstances, the DWBA should be replaced by a CC approach, in which inelastic excitations are treated to all orders. To avoid double counting of the effect of the inelastic channels on the elastic scattering, in the $\mathrm{CC}$ calculations the parameters of the monopole interaction have to be modified in

\footnotetext{
${ }^{1}$ The factor $\sqrt{2 I_{f}+1}$ makes the reduced matrix element invariant under $I_{i} \leftrightarrow I_{f}$ interchange, in agreement with the FRESCO convention.
} 


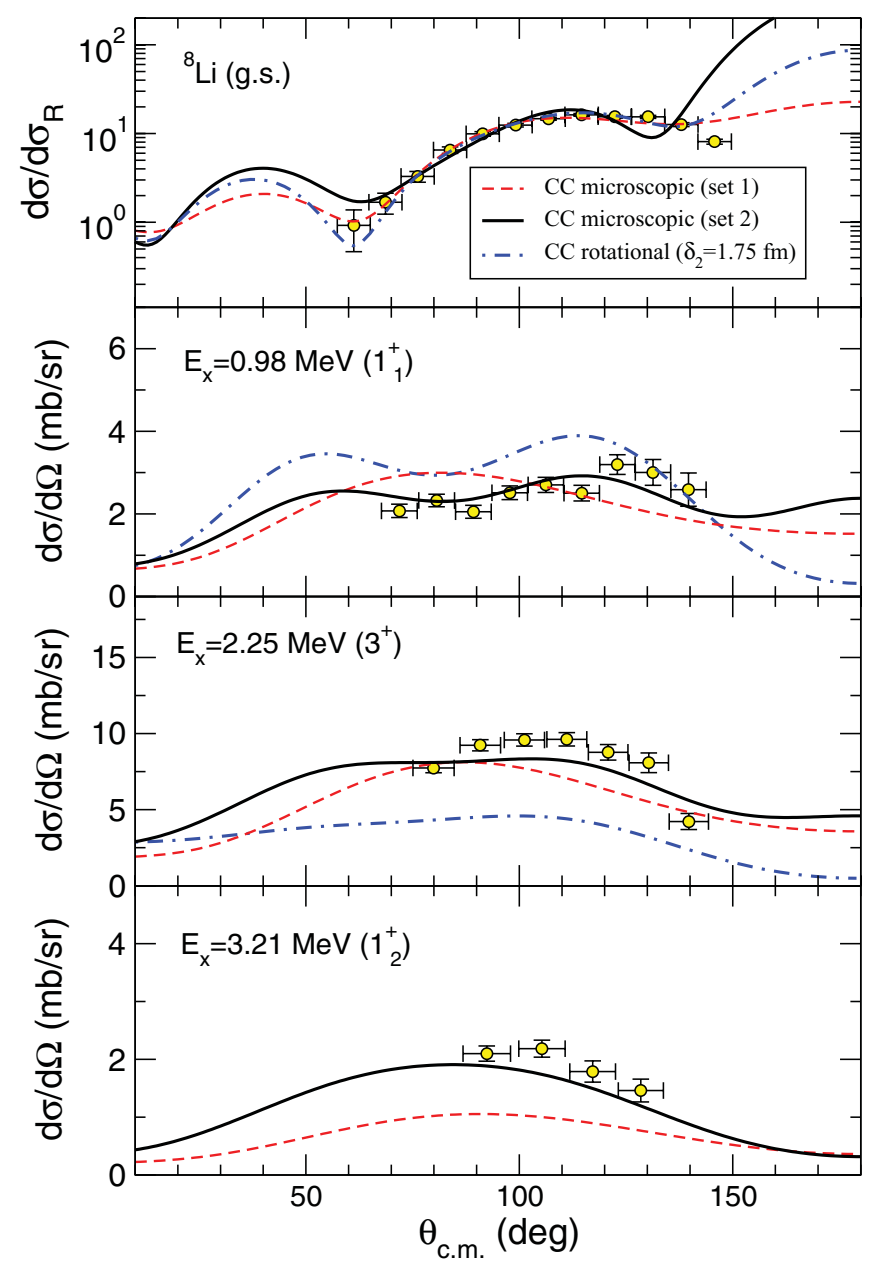

FIG. 7. (Color online) Coupled-channels calculations for $d+{ }^{8} \mathrm{Li}$ elastic scattering and inelastic scattering, leading to the low-lying excited states of ${ }^{8} \mathrm{Li}$. The solid and dashed lines are the CC calculations with two different deuteron potentials. The dotted-dashed line is the CC calculation assuming a rotational model for the ${ }^{8} \mathrm{Li}$ states, with an intrinsic quadrupole deformation length of $\delta_{2}=$ $1.75 \mathrm{fm}$.

order to restore the description of the elastic data. Thus, the parameters of the Woods-Saxon potential as well as those of the spin-orbit interaction were varied in order to minimize the $\chi^{2}$ resulting from the fit of the ground state and the $1_{1}^{+}$and $3^{+}$ excited states.

The calculated angular distributions are shown in Fig. 7 with dashed lines and the corresponding parameters are given in Table I CC (set 1). Compared to the DWBA results, these $\mathrm{CC}$ calculations show a better agreement with the data. This result confirms the importance of higher order effects, not included in the DWBA. However, the shape of the $1_{1}^{+}$angular distribution is not well described and the cross section for the $3^{+}$state is somewhat underestimated.

One can improve further the agreement with the inelastic cross sections by increasing arbitrarily the errors for the elastic data, in order to diminish its contribution to $\chi^{2}$. One of these calculations is illustrated in Fig. 7 by the solid line and the parameters are listed in Table I CC (set 2). It can be seen that the agreement is significantly improved for the inelastic channels, but at the expense of deteriorating the agreement with the elastic cross section. The impossibility of describing simultaneously the elastic and inelastic channels with the same degree of accuracy may be an indication for the presence of additional reaction mechanisms, besides the pure $\lambda=2$ quadrupole excitation considered here. A possible contribution would come from deuteron-target interactions with $\lambda=1$ and positive parity, such as spin-orbit forces, which could reorient the internal spin of the ${ }^{8} \mathrm{Li}$ nucleus $(S=1)$, while keeping its orbital angular momentum unaffected; this possibility is supported by the fact that the $1^{+} \rightarrow 2^{+} \gamma$-ray transition is dominated by $M 1$. A similar conclusion was achieved by Smith et al. [32] in their analysis of the ${ }^{12} \mathrm{C}\left({ }^{8} \mathrm{Li}^{8}{ }^{8} \mathrm{Li}^{\prime}\right){ }^{12} \mathrm{C}$ reaction. As a matter of fact, they needed a value for $B\left(E 2 ; 2^{+} \rightarrow 1^{+}\right)$ about an order of magnitude larger than the prediction of microscopic calculations. Due to the lack of knowledge of a realistic prescription for the form factors describing these $\lambda=1$ terms, we have not attempted to estimate quantitatively the contribution of this mechanism.

Finally, we note that nowhere in this analysis does one have to assume a rotational model for the ${ }^{8} \mathrm{Li}$ spectrum. This is in contrast to previous analyses of inelastic scattering of ${ }^{8} \mathrm{Li}$ [32]. To check the adequacy of the rotational picture for the ${ }^{8} \mathrm{Li}$ nucleus we have performed a new CC calculation assuming that the observed states belong to a $K=1^{+}$band. The central part of the projectile-target interaction is again described with the DF potential supplemented with a phenomenological imaginary part of a Woods-Saxon derivative shape and a spin-orbit potential. The coupling potentials are obtained by deforming the central interaction using the intrinsic deformation length $\delta_{2}=1.75 \mathrm{fm}$ [32]. The normalization of the DF potential as well as the parameters of the imaginary potential were adjusted to reproduce simultaneously the elastic and inelastic channels. The result of this fit is shown by the dot-dashed lines in Fig. 7. Note that the $1_{2}^{+}$state has not been included in these CC calculations since, strictly, the rotational model would predict only one $1^{+}$state. The elastic angular distribution is very well reproduced, but not the inelastic channels. In particular, the $2.25-\mathrm{MeV}$ state is underestimated by $50 \%$, whereas the cross section for the first excited state is overestimated. These results suggest that the rotational picture is inadequate to describe the couplings between the ${ }^{8} \mathrm{Li}$ states.

\section{Analysis of the cross sections}

The ${ }^{2} \mathrm{H}\left({ }^{8} \mathrm{Li}, p\right)^{9} \mathrm{Li}$ reaction corresponds to a $(d, p)$ reaction on ${ }^{8} \mathrm{Li}$. The excitation energy spectrum of the outgoing protons [Fig. 5(a)] exhibits three bumps corresponding to the population of the ${ }^{9} \mathrm{Li}$ ground state $\left(3 / 2^{-}\right)$and the excited states at $2.691 \mathrm{MeV}\left(1 / 2^{-}\right)$and $4.296 \mathrm{MeV}$ (with tentative assignment of $5 / 2^{-}$).

The measured angular distributions have been compared with DWBA calculations, under the assumption that these states are populated by means of a one-neutron direct transfer mechanism. In DWBA, the transition amplitude involves a matrix element of a transition operator between initial (deuteron) and final (proton) distorted waves. By using the post representation, the transition operator has the form 
$V_{[\mathrm{n}-\mathrm{p}]}+U_{\left[\mathrm{p}-{ }^{8} \mathrm{Li}\right]}-U_{\left[\mathrm{p}-{ }^{9} \mathrm{Li}\right]}$, where $V_{[\mathrm{n}-\mathrm{p}]}$ is the deuteron binding potential and $U_{\left[\mathrm{p}-{ }^{8} \mathrm{Li}\right]}$ and $U_{\left[\mathrm{p}-{ }^{9} \mathrm{Li}\right]}$ are effective interactions (complex in general) for the $p+{ }^{8} \mathrm{Li}$ and $p+{ }^{9} \mathrm{Li}$ systems. For the deuteron potential, we consider the potentials F1 and F2 found in Sec. III A. For the outgoing channel, due to the lack of data for the $p+{ }^{9} \mathrm{Li}$ reaction, we have used several prescriptions taken from the literature, labeled here P1 [33], P2 [30], and P3 [34]. The same potentials were used for the $p+{ }^{8} \mathrm{Li}$ interaction appearing in the remnant term of the transition potential, but with the radius suitably scaled to account for the mass difference.

The structure of the initial and final nuclei enters in the DWBA calculation through the overlap functions $\langle d \mid p\rangle$ and $\left\langle{ }^{9} \mathrm{Li} \mid{ }^{8} \mathrm{Li}\right\rangle$. The norm of these overlaps are, by definition, the spectroscopic factors. In principle, the calculation of the overlap functions requires knowledge of the many-body wave functions for the nuclei entering the overlap. Due to the complexity of this approach, it has become customary for many years to approximate the overlap function by the product of a unit-normalized single-particle wave function times a spectroscopic amplitude. The former is typically calculated by solving a one-body Schrödinger equation with a meanfield potential (typically a Woods-Saxon) with the quantum numbers and the separation energy of the removed nucleon. Spectroscopic factors are then obtained by normalizing the DWBA calculation to the experimental data. This procedure has been questioned in a number of works (see, e.g., $[35,36]$ ). The reason is that many transfer reactions are actually sensitive only to the tail of the overlap function (i.e., they are said to be peripheral). This overlap function is proportional at large distances to the Whittaker function, so what one actually probes in this peripheral reactions is the factor multiplying this Whittaker function, known as the asymptotic normalization coefficient (ANC), rather than the spectroscopic factor. If the overlap function is approximated by a single-particle wave function, then the ANC $\left(C_{l j}\right)$ is just the product of the spectroscopic amplitude $\left(A_{l j}\right)$ times the single-particle ANC $\left(b_{l j}\right)$. Since the latter depends on the choice of the mean-field potential, the spectroscopic factor determined by this procedure can be strongly model dependent. By contrast, if the process in peripheral, the ANC remains almost constant under moderate changes of the single-particle potential.

In the present case, we have found that the transfer cross section is sensitive to the $\left\langle{ }^{9} \mathrm{Li} \mid{ }^{8} \mathrm{Li}\right\rangle$ overlap for distances beyond $\approx 4 \mathrm{fm}$ and hence the peripherality condition is rather well fulfilled. Therefore, we will rely our analysis on the ANCs. However, for a comparison with previous works, spectroscopic factors will be also provided.

The $\langle d \mid p\rangle$ overlap was generated with a proton-neutron Gaussian potential $V_{p n}(r)=-72.15 \exp \left[-(r / 1.484)^{2}\right] \mathrm{MeV}$. The calculated overlap function has an ANC of $C=$ $0.87 \mathrm{fm}^{-1}$, in good agreement with the experimental value $0.8781(44) \mathrm{fm}^{-1}$ [37]. The $\left\langle{ }^{9} \mathrm{Li} \mid{ }^{8} \mathrm{Li}\right\rangle$ overlaps have been approximated by a single-particle wave function multiplied by the corresponding shell-model spectroscopic amplitudes. The latter were calculated with the $\mathrm{NN}$ effective interaction of Warburton and Brown [38], using the code OXBASH [39]. The single-particle wave functions were calculated with a Woods-Saxon potential with reduced radius $r_{0}=1.25 \mathrm{fm}$ and diffuseness $a=0.70 \mathrm{fm}$. The depth of the Woods-Saxon potential was adjusted to reproduce the experimental neutron separation energy. The ${ }^{9} \mathrm{Li}(4.3 \mathrm{MeV})$ state, which lies above the ${ }^{8} \mathrm{Li}$ (g.s.) $+n$ threshold, was interpreted as a single-particle resonance and described with a continuum bin. In this case the potential depth was adjusted to produce a resonance at the appropriate neutron- ${ }^{8} \mathrm{Li}$ relative energy $\left(\varepsilon_{\text {rel }}=\right.$ $0.23 \mathrm{MeV})$. The corresponding ANC can be obtained by multiplying the spectroscopic amplitude by the single-particle ANC. The calculated spectroscopic amplitudes and ANCs are shown in Table II. For the $\left\langle\left.{ }^{9} \mathrm{Li}(\right.$ g.s. $)\right|^{8} \mathrm{Li}($ g.s. $\left.)\right\rangle$ case, we include also the value from a variational Monte Carlo (VMC) calculation reported in Ref. [40]. In both cases, the predicted $p_{1 / 2}$ ANC is very small. For the $p_{3 / 2}$ configuration, both calculations are in good agreement, with the VMC value being smaller by about $8 \%$.

The results of these DWBA calculations are compared in Fig. 8 with the data. The lines correspond to different combinations of the deuteron $(\mathrm{F} 1, \mathrm{~F} 2)$ and proton $(\mathrm{P} 1, \mathrm{P} 2$, P3) potentials. For the transfer to ${ }^{9} \mathrm{Li}$ (g.s.) the set F1-P1 fails to describe correctly the shape of the oscillations of the data. Furthermore, in order to reproduce the magnitude of the data at forward angles, one needs a very small ANC $\left(C_{p 32}=0.79\right.$ $\mathrm{fm}^{-1 / 2}$ ), significantly smaller than the theoretical values (from either the shell model or the VMC method). When converted to a spectroscopic factor, using the single-particle ANC, it gives $S=0.31$. For other choices of the proton potential the calculation gave also the wrong shape of the angular distribution and very small spectroscopic factors (or ANCs). Therefore, we conclude that the deuteron potential F1 is not adequate to describe the present $(d, p)$ data. A similar conclusion has been reported by Powell et al. [30], who found that the deuteron potential that best describes the $(d, p)$

TABLE II. Shell-model spectroscopic amplitudes and ANCs $\left(\right.$ in $\mathrm{fm}^{-1 / 2}$ ) for ${ }^{9} \mathrm{Li} \rightarrow{ }^{8} \mathrm{Li}+1 n$. The ANCs labeled WBT have been computed by multiplying the shell-model amplitudes by the single-particle ANC $\left(b_{l j}\right)$. The ANCs labeled VMC correspond to the variational Monte Carlo calculations of Ref. [40]. See text for details.

\begin{tabular}{|c|c|c|c|c|c|c|c|}
\hline${ }^{9} \mathrm{Li}$ state & ${ }^{8} \mathrm{Li}$ state & \multicolumn{2}{|c|}{$A_{l j}(\mathrm{WBT})$} & \multicolumn{2}{|c|}{$C_{l j}(\mathrm{WBT})^{\mathrm{a}}$} & \multicolumn{2}{|c|}{$C_{l j}(\mathrm{VMC})$} \\
\hline $1 / 2^{-}$ & $2^{+}$ & -0.491 & - & 0.298 & - & & \\
\hline $5 / 2^{-}$ & $2^{+}$ & -0.451 & -0.718 & & & & \\
\hline
\end{tabular}

${ }^{\mathrm{a}} C_{l j}=A_{l j} \times b_{l j}$, where $A_{l j}$ is the shell-model spectroscopic amplitude and $b_{l j}$ is the single-particle ANC. 


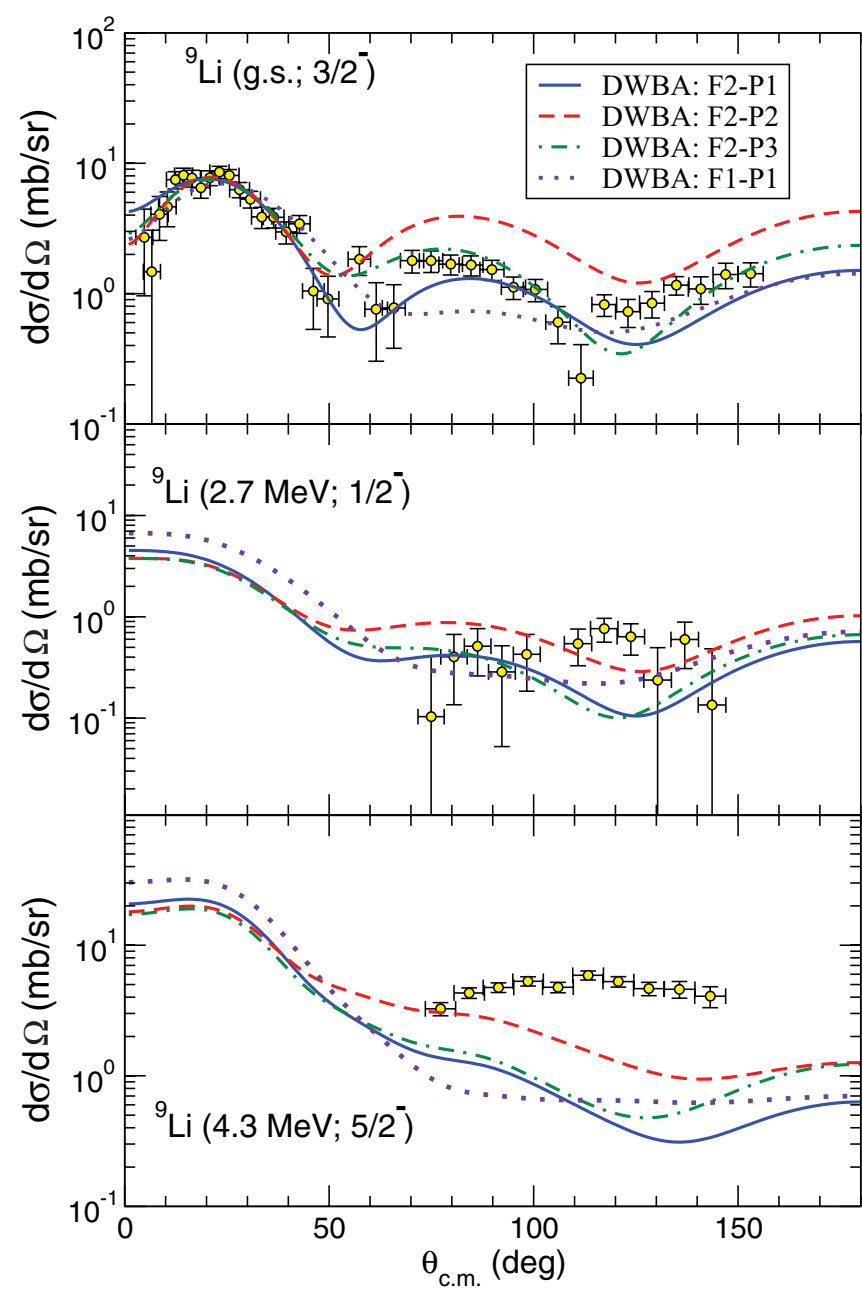

FIG. 8. (Color online) Experimental angular distributions for the ${ }^{2} \mathrm{H}\left({ }^{8} \mathrm{Li}, p\right) X$ reaction populating the first three states in ${ }^{9} \mathrm{Li}$ and results from DWBA calculations. The solid, dashed, and dotted-dashed lines correspond to the deuteron/proton potentials F2-P1, F2-P2, and F2P3, respectively. For the ${ }^{9} \mathrm{Li}$ g.s. (upper panel), the calculations have been normalized to reproduce the cross sections at forward angles. For the excited states, the calculations use shell-model spectroscopic amplitudes obtained with the WBT interaction and listed in Table II.

data does not reproduce the elastic data. The other three curves shown in Fig. 8 correspond to the DWBA calculations based on the deuteron potential F2, which uses the same imaginary radius as that used by Powell et al. The three proton potentials reproduce reasonably well the shape of data in the whole angular range. In order to extract the relevant ANC, the calculations have been normalized to reproduce the smaller angles, giving rise to the values $C_{p 32}=0.98,1.19$, and $1.10 \mathrm{fm}^{-1 / 2}$ for P1, P2, and P3, respectively. These ANCs agree very well with the VMC values from Ref. [40] listed in Table II. When converted to spectroscopic factors this gives $S_{p 32}=0.46,0.68$, and 0.58 , respectively. For the two excited states, the lack of experimental data at the forward angles prevented us from attempting the extraction of the spectroscopic factors by normalizing the calculation to the data.
The values extracted for the $\left(d, p_{0}\right)$ spectroscopic factor are somewhat smaller than the shell model (WBT) prediction $\left(S_{p 32}=0.73\right)$, but they agree reasonably well with those reported from other transfer reactions. For example, Kanungo et al. [16] found $S=0.59$ and 0.65 from $d\left({ }^{9} \mathrm{Li}, t\right)$ and Guimarães et al. [41] give $S=0.62(13)$ from ${ }^{9} \mathrm{Be}\left({ }^{8} \mathrm{Li},{ }^{9} \mathrm{Li}\right){ }^{8} \mathrm{Be}$. Of particular interest is the comparison with the results of Li et al. [42] and Guo et al. [43] since these works are based on the analysis of the same reaction, ${ }^{8} \mathrm{Li}(d, p){ }^{9} \mathrm{Li}$, at a somewhat higher energy $\left(E_{d}=9.75 \mathrm{MeV}\right)$. Li et al. [42] quote the value $S=0.68(14)$, obtained with the standard bound-state potential parameters $\left(r_{0}=1.25 \mathrm{fm}, a_{0}=\right.$ $0.65 \mathrm{fm})$. Guo et al. [43] extract the average ANC $C_{p 32}=$ $1.15 \mathrm{fm}^{-1 / 2}$, which is consistent with our values. It is interesting to note that the deuteron optical potentials used in the DWBA calculations of Ref. [43] have also a large radius parameter, $r_{i} \approx 2 \mathrm{fm}$.

The extracted spectroscopic factors are significantly smaller than the values found in our previous analysis of the ${ }^{9} \mathrm{Li}(d, t)^{8} \mathrm{Li}$ reaction at $2.36 \mathrm{MeV} /$ nucleon [14], which were about a factor of 2 larger than the shell-model prediction. The reason for this discrepancy is still uncertain but we may speculate that it could be related to the choice of the optical potentials and/or to possible contributions of higher order effects, not included in the DWBA calculations of Ref. [14].

For the transfer leading to the first excited state of ${ }^{9} \mathrm{Li}$, the DWBA calculations performed with the shell-model spectroscopic factors reproduce well the magnitude of the data, but not the shape. In the case of the second excited state, the calculations clearly underestimate the experimentally obtained cross section. This might indicate that this state is populated by a different mechanism, such as proton evaporation following the formation of a compound nucleus. Higher order effects, not taken into account in the DWBA method, might also contribute to the observed discrepancy. Due to the lack of reliable data at small c.m. angles, we have not attempted to extract the spectroscopic factors for these two states.

\section{Analysis of the ${ }^{2} \mathrm{H}\left({ }^{8} \mathrm{Li},{ }^{3} \mathrm{H}\right){ }^{7} \mathrm{Li}$ cross sections}

The excitation energy spectrum of detected tritons [Fig. 5(c)] shows very clearly the population of the ${ }^{7} \mathrm{Li}$ ground state and the resonances at $4.65 \mathrm{MeV}\left(7 / 2^{-}\right), 6.60 \mathrm{MeV}$ $\left(5 / 2_{1}^{-}\right)$, and $7.45 \mathrm{MeV}\left(5 / 2_{2}^{-}\right)$. Due to the limited energy resolution, the ground state could not be separated from the first excited state of ${ }^{7} \mathrm{Li}\left(1 / 2^{-} ; E_{x}=0.448 \mathrm{MeV}\right)$, so the angular distribution extracted from this peak may contain contributions from both states.

The angular distributions for these states have been compared with DWBA calculations, by assuming a one-neutron stripping process. For the incoming channel (deuteron) potential we used the potential F1 from Sec. III A. In contrast to the $(d, p)$ case, for the $(d, t)$ channels the calculated distributions showed only minor differences between potentials F1 and F2. The core-core potential $\left(d+{ }^{7} \mathrm{Li}\right)$ was taken from the work of Avrigeanu et al. [24]. The triton- ${ }^{7} \mathrm{Li}$ optical potential, used to generate the distorted waves in the outgoing channel, was taken from Dixon and Edge [44]. The depths of the real, imaginary, 
TABLE III. Shell-model spectroscopic amplitudes and ANCs for the ${ }^{8} \mathrm{Li} \rightarrow{ }^{7} \mathrm{Li}+1 n$ decomposition.

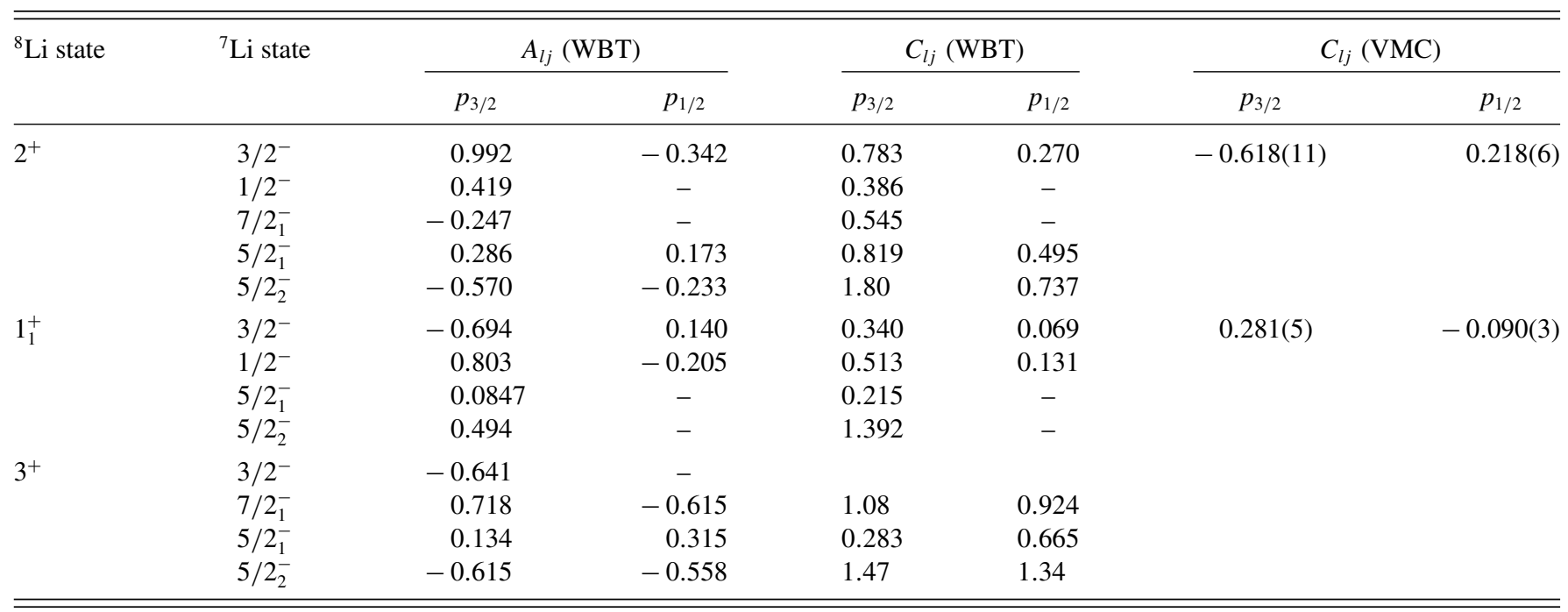

and spin-orbit parts were readjusted to reproduce the $t+{ }^{7} \mathrm{Li}$ elastic data of Ref. [45].

To generate the $\langle d \mid t\rangle$ overlap, we used a Woods-Saxon potential with geometry $R_{0}=1.5 \mathrm{fm}$ and $a=0.5 \mathrm{fm}$ and the depth adjusted to provide the experimental separation energy $(6.257 \mathrm{MeV})$. The single-particle wave function is multiplied by the spectroscopic amplitude 1.225, which gives an ANC of $C_{1 s}=2.01 \mathrm{fm}^{-1 / 2}$, in good agreement with the value $C_{1 s}=$ 2.07(2) $\mathrm{fm}^{-1 / 2}$, extracted in Ref. [46] from the experimental vertex function.

The $\left\langle{ }^{8} \mathrm{Li} \mid{ }^{7} \mathrm{Li}\right\rangle$ overlaps were approximated by a singleparticle wave function multiplied by the corresponding spectroscopic amplitude. The former was calculated in a WoodsSaxon potential with parameters $r_{0}=1.25 \mathrm{fm}$ and diffuseness $a=0.70 \mathrm{fm}$, which reproduces well the shape and the position of the maximum of the $\left\langle{ }^{8} \mathrm{Li} \mid{ }^{7} \mathrm{Li}\right\rangle$ overlap calculated with the VMC method [40]. The spectroscopic factors were again obtained from shell-model calculations with the WBT interaction. The calculated values are listed in Table III. For the $\left\langle{ }^{8} \operatorname{Li}(\right.$ g.s. $\left.)\right|{ }^{7} \operatorname{Li}($ g.s. $\left.)\right\rangle$ and $\left\langle\left.{ }^{8} \operatorname{Li}\left(1_{1}^{+}\right)\right|^{7} \operatorname{Li}(\right.$ g.s. $\left.)\right\rangle$ overlaps, we include also the ANC values from Ref. [40]. These are systematically smaller, but consistent with those derived from shell-model values. Because of the lack of data at forward angles in this channel, we have not attempted to extract the spectroscopic factors (or ANCs) by comparing the data with the calculations. Instead, we have used the theoretical values to assess the consistency of the present data with these values.

The DWBA calculations are shown by dashed lines in Fig. 9, along with the experimental data from the present experiment. The contribution of the ${ }^{7} \mathrm{Li}$ first excited state (dotted line in top panel) is found to be negligible. The calculation reproduces the magnitude of the ${ }^{7} \mathrm{Li}$ (g.s.) distribution, but not the shape. For the other states, the calculations clearly underestimate the data. One of the reasons for reduced calculated cross sections is the small value of the spectroscopic factors predicted for ${ }^{8} \mathrm{Li}($ g.s. $) \rightarrow{ }^{7} \mathrm{Li}\left(7 / 2^{-}\right)+n$ and ${ }^{8} \operatorname{Li}($ g.s. $) \rightarrow{ }^{7} \operatorname{Li}\left(5 / 2^{-}\right)+n$ (see Table III). However, the calculated spectroscopic factors (see Table III) suggest that

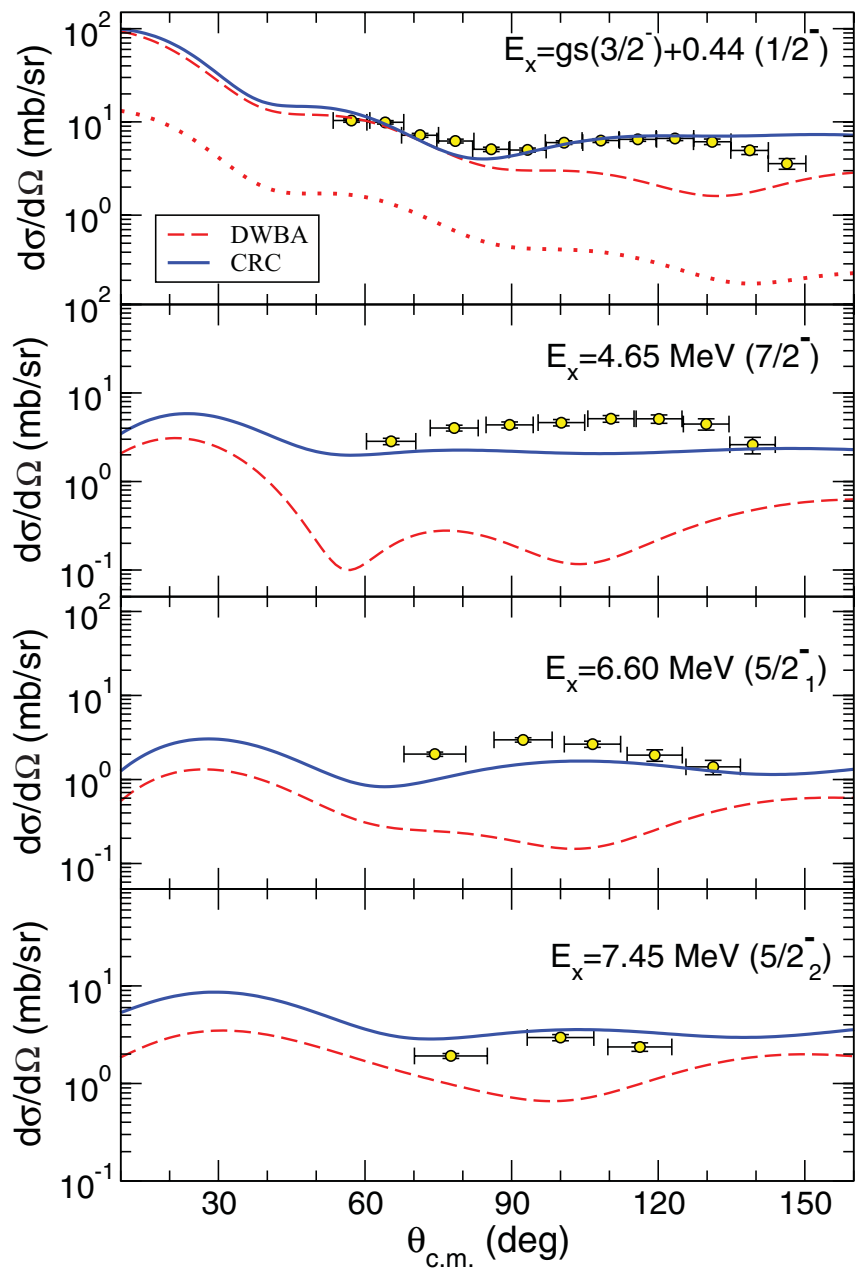

FIG. 9. (Color online) Measured angular distributions for the ${ }^{2} \mathrm{H}\left({ }^{8} \mathrm{Li}, t\right) \mathrm{X}$ cross sections, leading to several excited states of ${ }^{7} \mathrm{Li}$, compared with DWBA (dashed lines) and CRC (solid lines) calculations. The dotted line in the upper panel is the contribution of the $0.44-\mathrm{MeV}$ excited state in ${ }^{7} \mathrm{Li}$. 
these resonances have a large overlap with the excited states of ${ }^{8} \mathrm{Li}$. Since the latter are strongly populated in this process, one may speculate that an important mechanism populating the $7 / 2^{-}$and $5 / 2^{-}$resonances of ${ }^{7} \mathrm{Li}$ is by means of a multistep process through the excited states of ${ }^{8} \mathrm{Li}$. These higher order effects can be incorporated in the reaction formalism by treating the couplings in the incoming channel within the $\mathrm{CC}$ scheme, as done in Sec. III A.

In principle, in this approach the transfer can be still treated in first order (the so-called CCBA method). However, by doing so, we found that the calculated transfer cross section significantly exceeds the reaction cross section for several partial waves. This is a clear indication that the Born approximation is not adequate in this case. Consequently, we have to consider also higher order terms in the transfer couplings, thus performing a coupled-reaction-channels (CRC) calculation. To avoid double counting of the transfer couplings on the elastic scattering the depth of the imaginary part of the monopole interaction was reduced to $W_{d}=3 \mathrm{MeV}$. This value recovers the description of the elastic scattering once the effect of the transfer couplings are included. The CRC equations were solved iteratively, using Padé acceleration $[47,48]$ and nonorthogonality corrections.

The results of these CRC calculations are shown by solid lines in Fig. 9. The calculations reproduce the shape and magnitude of the ${ }^{7} \mathrm{Li}(\mathrm{g} . \mathrm{s} .+0.44)$ distribution very well. Furthermore, the magnitudes of $7 / 2^{-}, 5 / 2_{1}^{-}$, and $5 / 2_{2}^{-}$cross sections are also reasonably well reproduced. Given the significant number of physical ingredients of the CRC calculations (optical and binding potentials in the incoming and outgoing channels, deformation lengths, spectroscopic factors, etc,) it is difficult to trace back the source of the remaining discrepancies. Overall, given the absence of free parameters, we can conclude that the CRC calculations reproduce the whole data rather well, confirming the importance of multistep processes in this reaction.

As in the $(d, p)$ case, we found that the $(d, t)$ cross section is mostly sensitive to the tail of the $\left\langle{ }^{8} \mathrm{Li}||^{7} \mathrm{Li}\right\rangle$ overlap. This means that the magnitude of the calculated cross section is determined by the sum of the squares of the $p_{1 / 2}$ and $p_{3 / 2}$ ANCs. For the population of the ${ }^{7} \mathrm{Li}(\mathrm{g}$.s. $)$, by using the ANCs calculated from the shell-model spectroscopic factors, this quantity yields $C_{p 1 / 2}^{2}+C_{p 3 / 2}^{2}=0.686 \mathrm{fm}^{-1}$. This is larger than the VMC value, which gives $0.429 \mathrm{fm}^{-1}$. The latter is consistent with the value extracted experimentally by Trache et al. [49] from the ${ }^{13} \mathrm{C}\left({ }^{7} \mathrm{Li},{ }^{8} \mathrm{Li}\right){ }^{12} \mathrm{C}$ transfer reaction, $C_{p 1 / 2}^{2}+C_{p 3 / 2}^{2}=$ 0.432 (44) $\mathrm{fm}^{-1}$.

So, despite the reasonably good agreement with the data, one cannot rule out the presence of other mechanisms, such as compound nucleus formation and subsequent decay, that can contribute to the observed cross sections. The evaluation of these effects is, however, beyond the scope of this analysis.

\section{SUMMARY AND CONCLUSION}

The spectroscopic results obtained with an ${ }^{8} \mathrm{Li}$ beam represent a significant step forward in the data treatment and interpretation compared with the earlier experiment with a ${ }^{9} \mathrm{Li}$ beam. Absolute cross sections were deduced relatively straightforwardly, through the use of a detector constantly monitoring the beam current. In the former dataset, the absolute cross sections had to be calibrated with the help of elastic scattering data. Here, the data were obtained on an absolute scale not using any theoretical calculations, except the one for Rutherford scattering.

For the elastic channel the angular dependence of the cross section is well reproduced on an absolute scale using a doublefolding potential, generated with microscopic deuteron and ${ }^{8} \mathrm{Li}$ densities, and a phenomenological (Woods-Saxon) imaginary part. The deuteron energy spectrum shows also a strong coupling to the ${ }^{8} \mathrm{Li}$ excited states, particularly to the narrow $3^{+}$ resonance at $E_{x}=2.25 \mathrm{MeV}$. The angular distributions for the elastic and inelastic channels have been compared with DWBA and $\mathrm{CC}$ calculations, by using coupling potentials generated with microscopic transition densities [28] and assuming that the excited states are populated by quadrupole excitations.

The DWBA calculations, using a deuteron optical potential obtained from the fit of the elastic data, fail to describe the shape and magnitude of the inelastic angular distributions. Coupled-channels calculations, in which the coupling between the different states are included beyond the Born approximation, provide results in better agreement with the data, but some disagreement remains. The agreement can be improved by suitable changes of the deuteron optical model parameters, but at the expense of deteriorating the description of the elastic scattering data. These calculations suggest that other mechanisms, such as spin-flip $(\Delta S=1)$ transitions, might play a role in the description of the reaction dynamics. Moreover, CC calculations using a rotational model for the ${ }^{8} \mathrm{Li}$ states failed also to describe the data.

The proton spectrum shows the population of the ${ }^{9} \mathrm{Li}$ ground state, the excited state at $E_{x}=2.7 \mathrm{MeV}\left(1 / 2^{-}\right)$, and the resonance at $4.3 \mathrm{MeV}\left(5 / 2^{-}\right)$. The extracted angular distributions have been compared with DWBA calculations. The calculated cross sections have been found to be very sensitive to the deuteron potential. Furthermore, the deuteron potential that provides the best fit of the elastic cross section failed to reproduce the shape and magnitude of the data. In order to describe the $\left(d, p_{0}\right)$ data, a large value of the radius of the imaginary potential was required $\left(r_{i} \approx 2 \mathrm{fm}\right)$. Although we have not found an explanation for this empirical finding, it is consistent with other analyses of $(d, p)$ reactions on light targets at low energies $[30,43]$. The DWBA calculations based on this long-range imaginary potential reproduce reasonably well the shape of the experimental data, although for angles $\theta_{\text {c.m. }}>60^{\circ}$ the magnitude of the calculated cross section shows a significant dependence on the used optical model potentials. At forward angles, where the results are less sensitive to these potentials, the calculated cross section yields a spectroscopic factor consistent with the shell-model (WBT) prediction within $20 \%$. For the ${ }^{9} \mathrm{Li}$ excited states, due to the aforementioned optical model dependence, no attempt was made to extract the spectroscopic factors. Using the shell-model values, the calculations give the correct magnitude for the first excited state, but they clearly underestimate the data for the second excited state. This might be an indication of the importance of higher order effects, the inadequacy of the calculated 
spectroscopic factors, and/or a contribution from reactions forming compound nuclei.

For the $(d, t)$ channel, the data show the population of the ${ }^{7} \mathrm{Li}$ g.s. and first excited state $(0.44 \mathrm{MeV})$ and the three resonances at $E_{x}=4.65 \mathrm{MeV}\left(7 / 2^{-}\right), 6.60 \mathrm{MeV}\left(5 / 2_{1}^{-}\right)$, and $7.45 \mathrm{MeV}\left(5 / 2_{2}^{-}\right)$. DWBA calculations using theoretical spectroscopic factors failed to describe the angular distributions for these states. By contrast, CRC calculations, including multi-step processes through the excited states of ${ }^{8} \mathrm{Li}$ and higher orders in the transfer couplings accounted very well for the ${ }^{7} \mathrm{Li}$ ground state angular distribution and described also reasonably well the magnitude of the measured cross sections for the excited states. This result shows the importance of higher order effects in these low-energy reactions and points to the need for a careful examination of the validity of the DWBA in these processes.

The results demonstrate that transfer reactions remain a useful tool for investigating nuclear structure also with radioactive beams, in spite of additional obstacles such as unfavorable kinematics, loosely bound systems, and the low-energy beam available at REX-ISOLDE. However, only a very careful theoretical treatment allows a meaningful interpretation. Thus, the obtained results give confidence to investigate even more exotic nuclei where the excited states are not known and also to search for resonance states in unbound nuclei.

\section{ACKNOWLEDGMENTS}

The financial support of the Swedish Research Council for this work under Grant No. 2008-4158 is gratefully acknowledged, as well as support by the Lars Hierta Memorial Foundation. T. Nilsson acknowledges support through the Royal Swedish Academy of Sciences Research program by a grant from the Knut and Alice Wallenberg Foundation. This work is partially supported by the Spanish Ministerio de Ciencia e Innovación under Projects No. FPA2006-13807C02-01, No. FPA2009-08848, No. FPA2009-07653, No. FPA2009-07387, and No. FPA2010-17142 and by the European Union Sixth Framework through RII3-EURONS (Contract No. 506065).
[1] M. Huyse and R. Raabe, J. Phys. G: Nucl. Part. Phys. 38, 024001 (2011).

[2] G. C. Ball, L. Buchmann, B. Davids, R. Kanungo, C. Ruiz, and C. E. Svensson, J. Phys. G 38, 024003 (2011).

[3] J. R. Beene, D. W. Bardayan, A. G. Uribarri, C. J. Gross, K. L. Jones, J. F. Liang, W. Nazarewicz, D. W. Stracener, B. A. Tatum, and R. L. Varner, J. Phys. G 38, 024002 (2011).

[4] P. V. Duppen and K. Riisager, J. Phys. G 38, 024005 (2011).

[5] A. Navin, F. de Oliveira Santos, P. Roussel-Chomaz, and O. Sorlin, J. Phys. G 38, 024004 (2011).

[6] J. A. Clark, J. Phys. Conf. Ser. 267, 012002 (2011).

[7] A. H. Wuosmaa et al., Eur. Phys. J. Spec. Top. 150, 79 (2007).

[8] H. Lenske and G. Schrieder, Eur. Phys. J. A 2, 41 (1998).

[9] J. S. Winfield, W. N. Catford, and N. A. Orr, Nucl. Instrum. Methods A 396, 147 (1997).

[10] A. H. Wuosmaa et al., Phys. Rev. C 78, 041302 (2008).

[11] H. Jeppesen et al., Nucl. Phys. A 738, 511 (2004).

[12] H. B. Jeppesen et al., Nucl. Phys. A 748, 374 (2005).

[13] H. B. Jeppesen et al., Phys. Lett. B 642, 449 (2006).

[14] H. Jeppesen et al., Phys. Lett. B 635, 17 (2006).

[15] A. Koning, S. Hilaire, and M. Duijvestijn, in Proceedings of the International Conference on Nuclear Data for Science and Technology-ND2004, edited by R. C. Haight, M. B. Chadwick, T. Kawano, and P. Talou, AIP Vol. 769, 1154 (2005).

[16] R. Kanungo et al., Phys. Lett. B 660, 26 (2008).

[17] E. Kugler, Hyperfine Interact. 129, 23 (2000).

[18] O. Kester et al., Nucl. Instrum. Methods B 204, 20 (2003).

[19] F. Wenander, Nucl. Phys. A 701, 528 (2002).

[20] U. C. Bergmann, H. O. U. Fynbo, and O. Tengblad, Nucl. Instrum. Methods A 515, 657 (2003).

[21] D. R. Tilley, C. M. Cheves, J. L. Godwin, G. M. Hale, H. M. Hofmann, J. H. Kelley, C. G. Sheu, and H. R. Weller, Nucl. Phys. A 708, 3 (2002).

[22] D. R. Tilley, J. H. Kelley, J. L. Godwin, D. J. Millener, J. E. Purcell, C. G. Sheu, and H. R. Weller, Nucl. Phys. A 745, 155 (2004).
[23] I. J. Thompson, Comput. Phys. Rep. 7, 167 (1988).

[24] M. Avrigeanu, W. von Oertzen, U. Fischer, and V. Avrigeanu, Nucl. Phys. A 759, 327 (2005).

[25] R. V. Reid, Ann. Phys. (NY) 50, 411 (1968).

[26] G. Satchler and W. Love, Phys. Rep. 55, 183 (1979).

[27] J. Cook, Nucl. Phys. A 382, 61 (1982).

[28] D. Baye, P. Descouvemont, and N. K. Timofeyuk, Nucl. Phys. A 577, 624 (1994).

[29] J. Cook, Comput. Phys. Commun. 25, 125 (1982).

[30] D. L. Powell, G. M. Crawley, B. V. N. Rao, and B. A. Robson, Nucl. Phys. A 147, 65 (1970).

[31] A. M. Kobos, B. A. Brown, R. Lindsay, and G. R. Satchler, Nucl. Phys. A 425, 205 (1984).

[32] R. J. Smith, J. J. Kolata, K. Lamkin, A. Morsad, F. D. Becchetti, J. A. Brown, W. Z. Liu, J. W. Janecke, D. A. Roberts, and R. E. Warner, Phys. Rev. C 43, 2346 (1991).

[33] B. A. Watson, P. P. Singh, and R. E. Segel, Phys. Rev. 182, 977 (1969).

[34] D. H. Loyd and W. Haeberli, Nucl. Phys. A 148, 236 (1970).

[35] H. M. Xu, C. A. Gagliardi, R. E. Tribble, A. M. Mukhamedzhanov, and N. K. Timofeyuk, Phys. Rev. Lett. 73, 2027 (1994).

[36] A. M. Mukhamedzhanov et al., Phys. Rev. C 56, 1302 (1997).

[37] I. Borbely, W. Gruebler, V. Konig, P. A. Schmelzbach, and A. M. Mukhamedzhanov, Phys. Lett. B 160, 17 (1985).

[38] E. K. Warburton and B. A. Brown, Phys. Rev. C 46, 923 (1992).

[39] B. A. Brown, A. Etchegoyen, and W. D. M. Rae, MSU-NSCL Report No. 524, 1985.

[40] K. M. Nollett and R. B. Wiringa, Phys. Rev. C 83, 041001(R) (2011).

[41] V. Guimarães et al., Phys. Rev. C 75, 054602 (2007).

[42] Z. H. Li et al., Phys. Rev. C 71, 052801 (2005).

[43] B. Guo, Z. H. Li, W. P. Liu, X. X. Bai, G. Lian, S. Q. Yan, B. X. Wang, S. Zeng, J. Su, and Y. Lu, Nucl. Phys. A 761, 162 (2005). 
[44] R. L. Dixon and R. D. Edge, Nucl. Phys. A 156, 33 (1970).

[45] B. Y. Guzhovskij, S. N. Abramovich, B. M. Dzyuba, A. G. Zvenigorodskij, S. V. Trusillo, G. N. Sleptsov, and V. N. Protopopov, Probl. Yad. Fiz. Kosm. Luchei 7, 41 (1977).

[46] N. K. Timofeyuk, Phys. Rev. C 81, 064306 (2010).
[47] P. Graves-Morris, Padé Approximants (Institute of Physics, London, 1973).

[48] P. Wynn, Numer. Math. 8, 264 (1966).

[49] L. Trache, A. Azhari, F. Carstoiu, H. L. Clark, C. A. Gagliardi, Y.-W. Lui, A. M. Mukhamedzhanov, X. Tang, N. Timofeyuk, and R. E. Tribble, Phys. Rev. C 67, 062801 (2003). 